\title{
USE OF TACHYCARDIA TEMPLATES FOR RECOGNITION OF RECURRENT MONOMORPHIC VENTRICULAR TACHYCARDIA
}

\author{
Robert D. Throne MSE, Janice M. Jenkins PhD, Stuart A Winston* DO, Lorenzo A. DiCarlo* MD
}

Department of Electrical Engineering and Computer Science, University of Michigan

-Electrophysiology Laboratory, Saint Joseph Mercy Hospital, Ann Arbor, Michigan

\begin{abstract}
Various template matching methods for discriminating ventricular tachycardia (VT) from sinus rhythm (SR) based on ventricular electrogram morphology have been proposed. In this study, we wished to determine whether the ventricular electrograms of recurrent monomorphic ventricular tachycardia having an identical 12 lead surface ECG configuration might be matched to a template constructed from a previous episode of the same VT. Templates constructed during monomorphic VT in 12 patients were examined using Correlation Waveform Analysis $(C W A)$ and Area of Difference $(A D)$. Using $C W A$ the VT templates correctly discriminated 902/936 (97\%) ventricular depolarizations and $A D$ correctly discriminated $932 / 936(99 \%)$ depolarizations when the same VT was reinduced.
\end{abstract}

\section{Introduction}

There are many proposed methods for differentiating sinus rhythm (SR) from ventricular tachycardia (VT) for use in implantable antitachycardia devices. Many early methods are based primarily on timing information implemented in the early hardware available in antitachycardia devices [110]. Departing from rate-based schemes, morphology differences between sinus rhythm and ventricular tachycardia depolarizations are being employed for more accurate discrimination. These include the probability density function (PDF) [11] [12], Amplitude Distribution Analysis (ADA) [10] [13], the Gradient Pattern Detection (GPD) algorithm [14-16], Area of Difference $(A D)$ [17-20], and Correlation Wavefrom Analysis (CWA) [13] [21].

While discrimination of SR from VT may be improved by analysis of the morphology of the ventricular electrogram, rate related bundle branch block may confuse these algorithms [22]. This is because template matching methods generally have a binary desicion, i.e. the waveform matches the template (the patient is in SR or a supraventricular tachycardia) or the waveform does not match (the patient is in VT). However, bundle branch block of supraventricular origin also constitutes an abnormal cardiac depolarization sequence and may be misclassified by template matching schemes. Correctly matching the VT template would not only confirm deviation from normal cardiac conduction but also provide confirmation of a morphological match to a previously induced VT. This ability to confirm the VT with a previously known morphology would allow monitoring the number of times an antitachycardia device delivered therapy for a known VT in addition to the total number of therapies delivered. Finally, in future antitachycardia devices utilizing both atrial and ventricular electrograms, ventricular tachycardia with 1:1 retrograde conduction may be more easily distinguished from sinus tachycardia with aberrant conduction if the ventricular template matches a previously known VT.

In this study we chose to use both correlation waveform analysis and the area of difference method, two previously proposed template matching techniques, for use in testing the possibility of matching templates constructed during monomorphic ventricular tachycardia with subsequent episodes of the same VT. Correlation waveform analysis computes the correlation coefficient, $\rho$, between a previously constructed ventricular depolarization template and the ventricular waveform under analysis, and yields a statistical measure of the similarilty between two waveforms [13]. The correlation coefficient produces a bounded performance measure between -1 and 1 indicating how well the template matches the depolarization under analysis and is independent of baseline changes and amplitude changes of the waveforms. The area of difference method computes the sum of the absolute value between template points and the corresponding points of the waveform under analysis. Area of difference is dependent on amplitude changes and is not independent of baseline changes. In addition, there is no error bound produced using this method.

\section{Methods and Materials}

Electrophysiology Study. Bipolar ventricular endocardial electrograms were recorded during elective clinical cardiac electrophysiology studies. Our patient population consisted of 9 men and 3 women. Patients were studied in a fasting postabsorptive state after sedation with $1-3 \mathrm{mg}$ of intravenous medazolam. After administering $1 \%$ lidocaine for local anesthesia, three 7 French side-arm sheaths (Cordis Corporation) were positioned in the right femoral vein using the Seldinger technique. Three 6 French quadrapolar electrode catheters with an interelectrode distance of $1 \mathrm{~cm}$ (USCI division, C. R. Bard Inc.) were introduced and advanced under fluoroscopic guidance to the high right atrium and right ventricular apex. Immediately before programmed stimulation a 12-lead electrocardiogram was 
recorded during sinus rythm. Ventricular electrograms were recorded on FM magnetic tape (Hewlett Packard Models $\$ 968$ and $\$ 964 A$ ) from distal bipolar endocardial electrodes positioned in the right ventricular apex using amplifiers with filter settings of 0.5 to $500 \mathrm{~Hz}$ (Siemens Mingograf$\eta$ or 1 to $500 \mathrm{~Hz}$ (Honeywell Electronics for Medicine). Tape speed was $3 \frac{3}{4}$ inches per second with a bandwidth of 0 to $1250 \mathrm{~Hz}$. All recordings were made with the patients lying supine. The recorded ventricular electrograms were subsequently digitized on a personal computer with an analog-to-digital system (Scientific Solutions, Inc.) at a sampling rate of $1000 \mathrm{~Hz}$. The programs for digitization and subsequent waveform analysis were written in $\mathrm{C}$ programming language and assembly language.

Methods of Analysis. Data sets typically consisted of four 15 second passages from each patient. Two separate passages were digitized from ventricular electrograms during sinus rhythm and a third passage was digitized from a segment of induced monomorphic ventricular tachycardia. A fourth passage was recorded and digitized from the reinduced ventricular tachycardia which appeared the same in $12 / 12$ surface leads as the initially induced VT. The VT template was constructed by averaging ventricular depolarizations and was chosen to include only the depolarization portion of the waveform. Both $C W A$ and $A D$ were then used to match the template with the subsequent induction and both passages of sinus rhythm.

Triggering. A software trigger was used for detection of waveforms. A best fit algorithm was used to provide the best alignment for each algorithm within an 11 millisecond window centered on the original trigger point as described previously [23]. The location of the best alignment for one algorithm did not necessarily coincide with the best alignment for another algorithm.

Correlation Waveform Analysis (CWA). The correlation coefficient, used by $C W A[13][21]$ is computed as

$$
\rho=\frac{\sum_{i=1}^{i=N}\left(t_{i}-\bar{t}\right)\left(s_{i}-\bar{s}\right)}{\sqrt{\sum_{i=1}^{i=N}\left(t_{i}-\bar{t}\right)^{2}} \sqrt{\sum_{i=1}^{i=N}\left(s_{i}-\bar{s}\right)^{2}}}
$$

where $\rho=$ the correlation coefficient, $N=$ the number of template points, $t_{i}=$ the template points, $s_{i}=$ the signal points under analysis, $\bar{t}=$ the average of the template points, and $\bar{s}=$ the average of the signal points. The formula for the correlation coefficient can equivalantly be rewritten as

$$
\rho=1-\frac{1}{2} \sum_{i=1}^{i=N}\left(\frac{t_{i}-\bar{t}}{\sqrt{\sum_{k=1}^{k=N}\left(t_{k}-\bar{t}\right)^{2}}}-\frac{s_{i}-\bar{s}}{\sqrt{\sum_{k=1}^{k=N}\left(s_{k}-\bar{s}\right)^{2}}}\right)^{2}
$$

Area of Difference (AD). The area of difference [17] [18] [20] directly measures the absolute difference in amplitude of sample points of the template and the waveform under analysis. Mathematically,

$$
A D=\sum_{i=1}^{i=N}\left|t_{i}-s_{i}\right|
$$

where $N=$ the number of template points, $t_{i}=$ the template points, and $s_{i}=$ the signal points under analysis. The area of difference is usually reported as a percentage change of the absolute deviation of the template points from the baseline, i.e.,

$$
\begin{gathered}
A D(\%)=\frac{\sum_{i=1}^{i=N}\left|t_{i}-s_{i}\right|}{\sum_{i=1}^{i=N}\left|t_{i}\right|} \times 100 \% \\
\text { Results }
\end{gathered}
$$

The results for each patient are shown in Table 1. For each patient, the number of incorrect classifications for each of the two methods is shown, as well as the total number of depolarizations processed for each patient. There was only one patient with misclassifications using $A D$ (4 depolarizations in total), while with $C W A$ there were 4 patients with misclassifications ( 32 depolarizations in total). In two patients there was only 1 depolarization misclassified, while for the other two patients 7 out of 71 and 23 out of 54 depolarizations were misclassified. The ranges and mean values for the patients processed are shown in Figure 1 for $C W A$ and in Figure 2 for $A D$. Results for each patient are displayed as a column located on the horizontal axis, while the ranges of correlation coefficient or area of difference are displayed along the vertical axis. The ranges of correlation coefficient or area of difference for the second VT induction is shown in white (with a black box at the mean), while the range for the two sinus rhythm passages are shown in black (with a white box at the mean).

Figure 3 show passages of sinus rhythm and VT with 1:1 retrograde conduction for patient 5 . Both passages are played at $50 \mathrm{~mm} / \mathrm{sec}$ and at identical gain settings. Both $C W A$ and $A D$ distinguish all VT from sinus rhythm depolarizations using the VT template.

\begin{tabular}{|c||c|c|c|}
\hline Patient & $\begin{array}{c}C W A \\
\text { Overlap }\end{array}$ & $\begin{array}{c}A D \\
\text { Overlap }\end{array}$ & $\begin{array}{c}\text { Total Number of } \\
\text { Depolarizations }\end{array}$ \\
\hline \hline 1 & 0 & 0 & 78 \\
\hline 2 & 1 & 0 & 88 \\
\hline 3 & 1 & 0 & 69 \\
\hline 4 & 0 & 0 & 80 \\
\hline 5 & 0 & 0 & 98 \\
\hline 6 & 0 & 0 & 68 \\
\hline 7 & 0 & 0 & 54 \\
\hline 8 & 0 & 0 & 76 \\
\hline 9 & 7 & 4 & 71 \\
\hline 10 & 0 & 0 & 60 \\
\hline 11 & 0 & 0 & 69 \\
\hline $6 *$ & 0 & 0 & 71 \\
\hline 12 & 23 & 0 & 54 \\
\hline
\end{tabular}

Table 1: Results using correlation waveform analysis $(C W A)$ and area of difference (AD). The number in columns 2 and $S$ indicate the number of depolarizations with overlapping values for $C W A$ and $A D$. 'Patient 6 was studied on two different days with two different VT morphologies. 


\section{PATIENT}

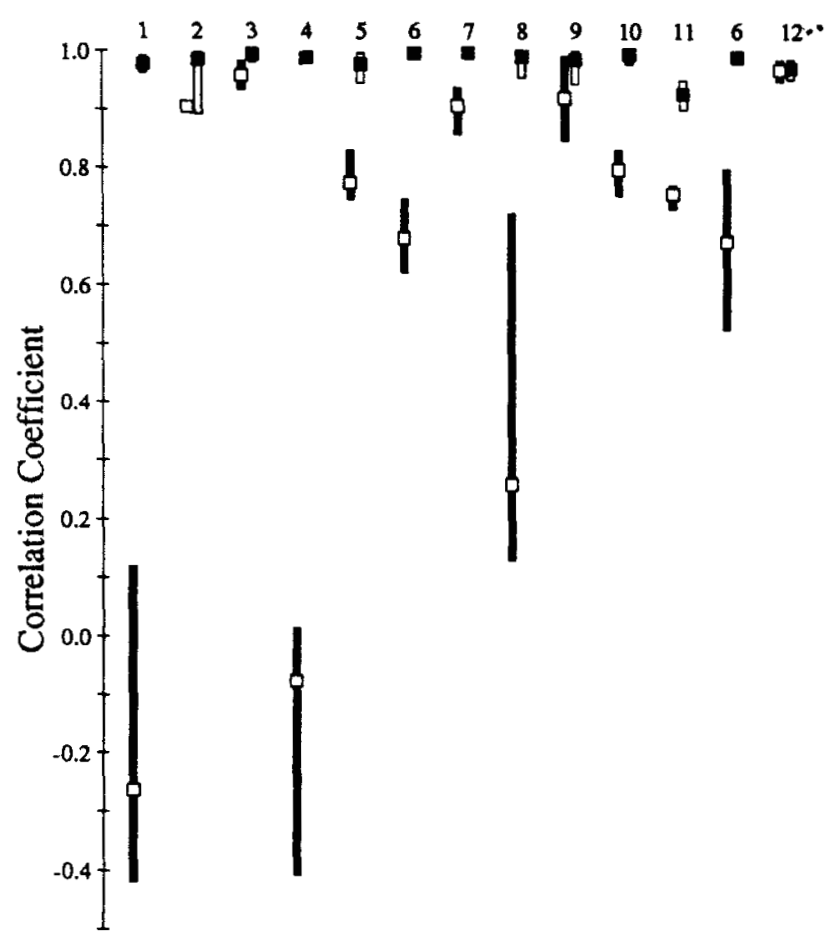

Figure 1: Results using CWA. Ranges of VT are in white while ranges for the $S R$ passages are in black.

Limitations. This study was done using acute leads positioned during electrophysiologic study and has not been tested or validated on chronic leads. The monomorphic VT was reinduced during a single electrophysiology study. Whether changes in heart substrate or structure may cause changes in the intracardiac VT morphology over time has not been determined.

Conclusion. We conclude that templates constructed from recurrent monomorphic ventricular tachycardia may be feasible for confirmation of the same ventricular tachycardia occurring at a later time. This may allow assessment

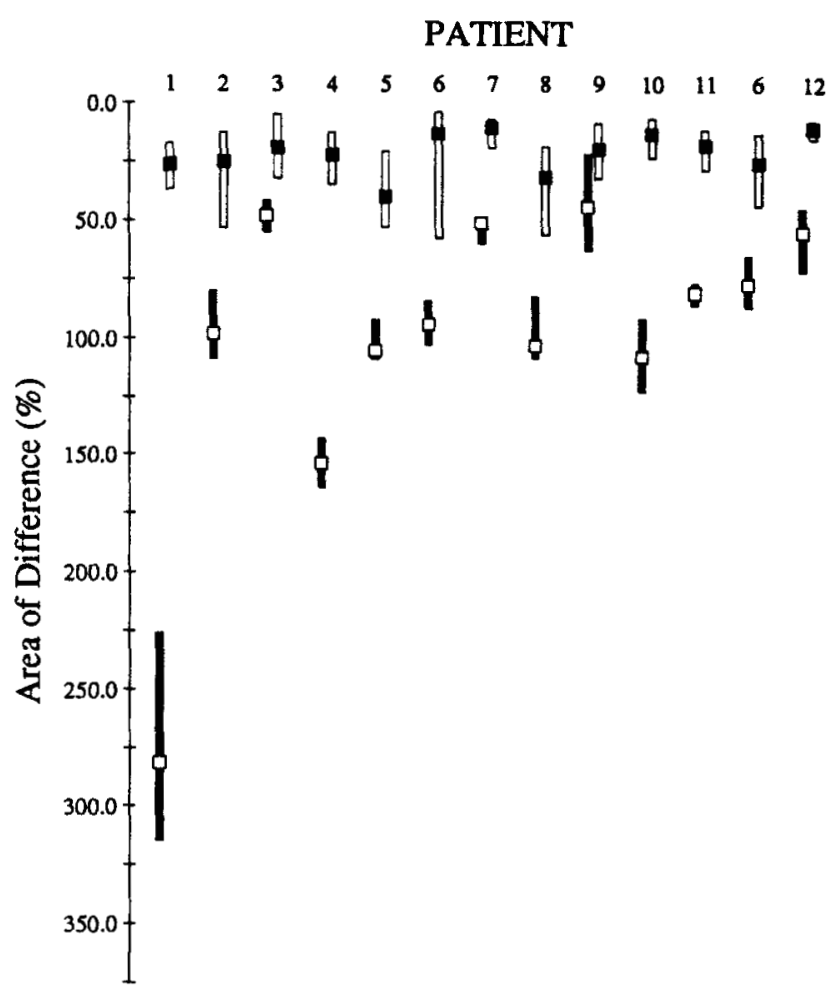

Figure 2: Results using $A D$. Ranges of VT are in white while ranges for the $S R$ passages are in black.

of the number of times a device has delivered therapy for a known VT in addition to the total number of therapies for cardiac rhythms which may have exceeded a rate criteria and/or mophology criteria. If further study shows this method to be successful, it may be desirable for future antitachycardia devices to be programmed to deliver therapies based on recognition of the morphology of the VT as well as its rate. In addition, VT templates may allow confirmation of the recurrence of known VT morphologies.

Acknowledgement. This work was partially supported by NSF grant EET-895125 and a grant by Medtronic, Inc.
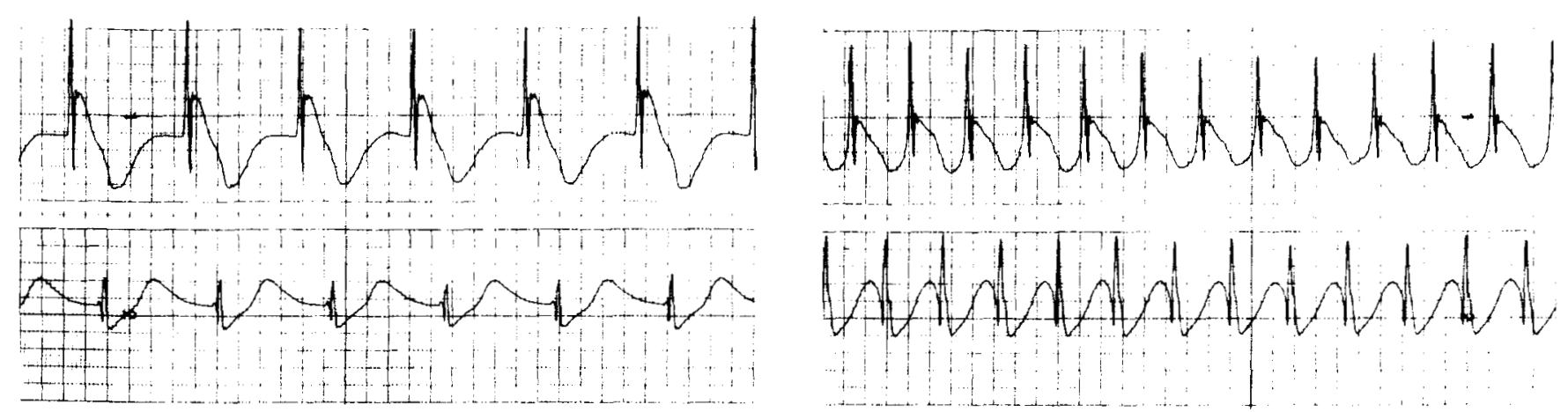

Figure 3: Sample tracing of intracardiac electrograms during sinus rhythm (left) and ventricular tachycardia (right). The top channel is the atrial signal, the lower is the ventricular signal. 


\section{References}

[1] J. D. Fisher, M. Goldstein, E. Ostrow, J. A. Matos, and S. G. Kim, Maximal rate of tachycardia development: Sinus tachycardia with sudden exercise vs. spontaneous ventricular tachycardia, $P A C E, 6$ :221228. 1983.

[2] A. Geibel, M. Zehender, and P. Brugada, Changes in cycle length at the onset of sustained tachycardias - Importance for antitachycardia pacing, American Heart Journal, 588-592. 1988.

(3) M. Fromer, T. Kus, M. Dubuc, R. Nadeau, and M. Shenasa, Oscillation of ventricular tachycardia cycles length, PACE, 10:451. 1987. (Abstract)

[4] A. W. Nathan, J. E. Creamer, D. W. Davies, and J. E. Camm, Clinical experience with a new versatile, software based, tachycardia reversion pacemaker, Journal of the American College of Cardiology, 7:184A. 1986. (Abstract)

[5] W. H. Olson and G. H. Bardy, Cycle length and morphology at the onset of spontaneous ventricular tachycardia and fibrillation, PACE, 9:284. 1986. (Abstract)

[6] W. Olson, G. Bardy, R. Mehra, C. Almquist, and R. Biallas, Comparison of different onset and stability algorithms for detection of spontaneous ventricular arrythmias, $P A C E, 10: 439$. 1987. (Abstract)

[7] W. Olson, G. Bardy, R. Mehra, C. Almquist, and R. Biallas, Onset and stability for ventricular tachycardia detection in an implantable pacer-cardioverterdefibrillator, IEEE Computers in Cardiology, pp. 167170. Leuven, Belgium: IEEE Computer Society. 1987.

[8] G. Tomaselli, M. Scheinman, and J. Griffin, The utility of timing algorithms for distinguishing ventricular from supraventricular tachycardias, $P A C E, 10: 415$. 1987. (Abstract)

[9] J. Warren and R. O. Martin, Clinical evaluation of automatic tachycardia diagnosis by an implanted device, Cardiostim 86, 4:16. 1986. (Abstract)

[10] K.L. Ripley, T.E. Bump, and R.C. Arzbaecher, Evaluation of techniques for recognition of ventricular arrhythmias by implanted devices, IEEE Transactions on Biomedical Engineering, 36:618-624. 1989.

[11] M. Mirowski, M. M. Mower, and P. R. Reid, The automatic implantable defibrilator, American Heart Journal, 100:1089-1092. 1980.

[12] M. Mirowski, M. M. Mower, P. R. Reid, L. Watkins, and $A$. Langer, The automatic implantable defibrillator: New modality for treatment of life-threatening ventricular arrhythmias, $P A C E$, 5:384-401. 1982.
[13] D. Lin, L. A. DiCarlo, and J. M. Jenkins, Identification of ventricular tachycardia using intracavitary ventricular electrograms: analysis of time and frequency domain patterns, PACE, 11:1592-1606. 1988.

[14] D.W. Davies, R.J. Wainwright, M. Tooley, D. Loyd, and A.J. Camm, Electrogram recognition by digital analysis: relevance to pacemaker arrhythmia control, Journal of the American College of Cardiology, 5:507. 1985. (Abstract)

[15] D. W. Davies, R. J. Wainwright, M. A. Tooley, D. Lloyd, A. W. Nathan, R. A. J. Spurrell, and A. J. Camm, Detection of pathological tachycardia by analysis of electrogram morphology, $P A C E, 9: 200-208$. 1986.

[16] D.W. Davies, M.A. Tooley, T. Cochrane, A.W. Nathan, R.J. Wainwright, and A.J. Camm, Real-time tachycardia diagnosis using morphological analysis of electrograms, PACE 10:998. 1987. (Abstract)

[17] J. L. Langberg, W. J. Gibb, D. M. Auslander, and J. C. Griffin, Identification of ventricular tachycardia with use of the morphology of the endocardial electrogram, Circulation, 77:1363-1369. 1988.

[18] G. F. Tomaselli, A. P. Nielsen, W. L. Finke, L. Singupta, J. C. Clarck, and J. C. Griffin, Morphologic differences of the endocardial electrogram in beats of sinus and ventricular origin, $P A C E, 11: 254-262.1988$.

[19] J. C. Griffin, J. Langberg, G. Tomaselli, and W. Gibb, Endocardial electrogram morphology: a unique characteristic of cardiac rhythm ?, Cardiostim 86, 4:15. 1986. (Abstract)

[20] G. Tomaselli, W.J. Gibb, J.J. Langberg, M.C. Chin, and J.C. Griffin, In vivo testing of a morphology based approach to cardiac rhythm identification, Circulation, 76:1116. 1987. (Abstract)

[21] L. DiCarlo, D. Lin, and J. Jenkins, Analysis of time and frequency domain patterns to distinguish ventricular tachycardia from sinus rhythm using endocardial electrograms, Circulation, 76:280. 1987. (Abstact)

[22] R. Throne, J. Jenkins, S. Winston, and L. DiCarlo, Ventricular tachycardia versus supraventricular tachycardia with aberration: An analysis of bundle branch dharacteristics using intracardiac electrograms, PACE, 12:662. April 1989. (Abstract)

[23] R.D. Throne, J.M. Jenkins, S.A. Winston, C.J. Mays, and L.A. DiCarlo, Discrimination of retrograde from anterograde atrial activation using intracardiac electrogram waveform analysis, $P A C E, 12: 640$. October 1989. 